\title{
Quantum network recovery from multinode failure using network encoding with GHZ states on higher-order butterfly networks
}

\author{
M. G. Majumdar ${ }^{1}$ (D) S. S. Garani ${ }^{1}$
}

Received: 26 June 2021 / Accepted: 2 November 2021 / Published online: 17 November 2021

(c) The Author(s), under exclusive licence to Springer Science+Business Media, LLC, part of Springer Nature 2021

\section{Abstract}

We propose a protocol to transmit three quantum states crossly in a butterfly network with prior entanglement, in the form of GHZ states, between three senders. The proposed protocol requires only one qubit transmission or two classical bits transmission in each channel of the network. We generalise this protocol to higher number of qubits with multiqubit GHZ states towards quantum network operability using network coding with multiqubit GHZ states on higher-order butterfly networks.

Quantum correlations, particularly prior entanglement across quantum states, can be harnessed for transmitting more classical information through quantum communication links through teleportation and superdense coding schemes [1-5]. The physical realisation of quantum networked systems at atomic scale distances using such entangled quantum states is key towards realising high-throughput quantum communications at such scales. The ideas from classical network coding, such as coding over butterfly networks, can be naturally extended to the quantum case, mindful of the quantum no-go theorems [6,7]. In the butterfly network, two units of information can be sent crossly and the channels can transmit only one bit, with the bottleneck being the central channel in the network.

It was recently shown that perfect quantum state transmission is impossible in the butterfly network and the bottleneck, in the form of the central channel, cannot be resolved for a quantum network [6]. This was subsequently extended to different kinds of networks with quantum network coding [8]. Leung et al proposed network coding using shared entanglement between two parties [9] through quantum teleportation and superdense coding. Hayashi demonstrated the impossibility of transmitting quantum states over the butterfly network between two senders without prior entanglement [11]. In this letter, we have formulated a non-trivial extension of Hayashi's results to the

\footnotetext{
M. G. Majumdar mrittunjoyg@iisc.ac.in

S. S. Garani shayangs@iisc.ac.in

1 Indian Institute of Science, Bangalore, India
} 

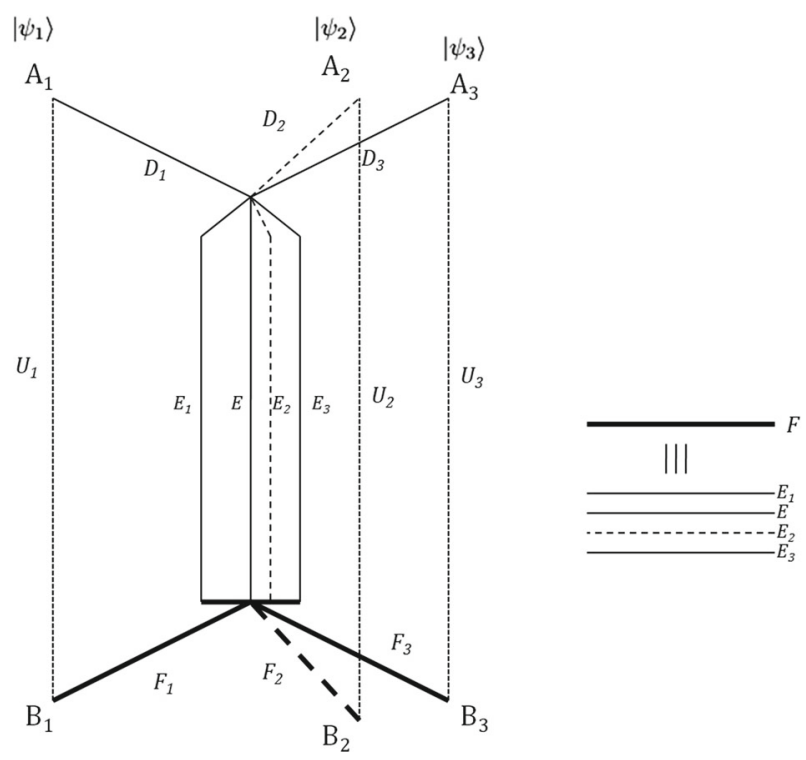

Fig. 1 Network for quantum butterfly network comprising of three users with three GHZ states and an arbitrary quantum state for each transmission, along with four central channels: $E=\left(X_{1}^{(a)} \oplus X_{2}^{(a)} \oplus\right.$ $\left.X_{3}^{(a)}, X_{1}^{(b)} \oplus X_{2}^{(b)} \oplus X_{3}^{(b)}\right), E_{1}=\left(X_{1}^{(a)} \oplus X_{2}^{(a)}, X_{1}^{(b)} \oplus X_{2}^{(b)}\right), E_{2}=\left(X_{2}^{(a)} \oplus X_{3}^{(a)}, X_{2}^{(b)} \oplus X_{3}^{(b)}\right)$ and $E_{3}=\left(X_{1}^{(a)} \oplus X_{3}^{(a)}, X_{1}^{(b)} \oplus X_{3}^{(b)}\right)$. The channels $U_{1}, U_{2}$ and $U_{3}$ are quantum channels, while $D_{1}, D_{2}$, $D_{3}, E, E_{1}, E_{2}, E_{3}, F_{1}, F_{2}$ and $F_{3}$ are classical channels carrying measurements values or their unique combinations

case of higher-order butterfly networks using GHZ states. This extension is useful to realise polygon tessellated higher-order butterfly networks for recovery of network operability post-detection of erased nodes.

Our protocol is based on a network with prior entanglement shared between three users. We fundamentally use teleportation for the proposed protocol (Fig. 1).

We assume that the three senders $A_{1}, A_{2}$ and $A_{3}$ share three copies of maximally entangled (GHZ) states: $\left|\phi_{i}\right\rangle=\frac{1}{\sqrt{2}}(|000\rangle+|111\rangle)_{(1, i),(2, i),(3, i)}$, where $i=1,2,3$, denotes the $i^{\text {th }}$ GHZ state, while the qubits with the same first index in the subscript belong to the same physical terminal. The senders prepare their states in: $\left|\psi_{j}\right\rangle=\alpha_{j}|0\rangle+\beta_{j}|1\rangle$, where $j=1,2,3$ denotes the $j^{\text {th }}$ user. In the first step, the sender $A_{i}$ performs a Bell state measurement $\left\{\phi_{+}, \phi_{-}, \psi_{+}, \psi_{-}\right\}$on the joint system $A_{i} \otimes A_{i, i}$. We can see the decomposition for $A_{i} \otimes A_{i, i} \otimes A_{(i+1) \bmod 3, i} \otimes A_{(i+2) \bmod 3, i}, i=1,2,3$ in Table 1 . Here $A_{i}$ represents the arbitrary prepared state, while $A_{i, i}, A_{(i+1) \bmod 3, i}$ and $A_{(i+2) \bmod 3, i}$ are qubits from the same GHZ state at three different physical terminals. After the first Bell state measurement, we undertake an additional measurement on the single qubit component of the GHZ state at one of the other two nodes. We denote this measurement as $X_{i}^{(b)}$, with the first measurement being tagged as $X_{i}^{(a)}$. In this measurement, we measure single qubits in the $| \pm\rangle=\frac{1}{\sqrt{2}}(|0\rangle \pm|1\rangle$ basis. The outstanding problem of the directionality of cycling in the aforementioned scheme can be resolved 
Table 1 Decomposition for $A_{i} \otimes A_{i, i} \otimes A_{(i+1) \bmod 3,1} \otimes$ $A_{(i+2) \bmod 3,1} \forall i=1,2,3$, and truth values as well as associated unitary transformations for higher-order butterfly network over three nodes. Here $B=$ $A_{(i+1) \bmod 3, i} \otimes A_{(i+2) \bmod 3, i}$

\begin{tabular}{lllll}
\hline$X_{i}^{(a)}$ & $X_{i}^{(b)}$ & $A_{i} \otimes A_{i, i}$ & $B$ & $U$ \\
\hline$(0,0)$ & $0 / 1$ & $\left|\psi_{+}\right\rangle$ & $\alpha_{1}|00\rangle+\beta_{1}|11\rangle$ & $I / \sigma_{z}$ \\
$(0,1)$ & $0 / 1$ & $\left|\psi_{-}\right\rangle$ & $\alpha_{1}|00\rangle-\beta_{1}|11\rangle$ & $\sigma_{z} / I$ \\
$(1,0)$ & $0 / 1$ & $\left|\phi_{+}\right\rangle$ & $\alpha_{1}|11\rangle+\beta_{1}|00\rangle$ & $\sigma_{x} / \sigma_{x} \sigma_{z}$ \\
$(1,1)$ & $0 / 1$ & $\left|\phi_{-}\right\rangle$ & $\alpha_{1}|11\rangle-\beta_{1}|00\rangle$ & $\sigma_{x} \sigma_{z} / \sigma_{x}$ \\
\hline
\end{tabular}

in terms of an activation variable $\mathcal{A}_{i}$ and cyclicity variable $\mathcal{C}_{i}$ for the $i^{\text {th }}$ node, which can be transported using the classical infrastructure of the scheme, specifically using $X_{i}^{(b)}$ in a time-sequenced manner. The activation variable denotes that the node has a state to transfer ( 0 for no state to transfer while 1 is for a state to transfer) while cyclicity variable denotes whether the node wants to transport clockwise (0) or anti-clockwise (1). Let us say there is a characteristic time $T$. In time $0<t \leq T$, the nodes transmit the measurement variables $\left(X_{i}^{(a)}, X_{i}^{(b)}\right)$. In time $T<t \leq 2 T$, the nodes transmit the activation variable over the $X_{i}^{(b)}$ channel. In time $2 T<t \leq 3 T$, we transmit the cyclicity variable. Using these units of information, the relevant quantum information is crossly transmitted across such a higher-order butterfly network.

The action of Bell state measurement leaves a state $U\left(X_{3}^{a}, X_{2}^{b}\right)^{-1}\left|\psi_{3}\right\rangle$ or $U\left(X_{2}^{a}, X_{3}^{b}\right)^{-1}$ $\left|\psi_{2}\right\rangle$ depending on a sequence of measurements at the first qubit. Then the first terminal applies $U\left(X_{1}^{a}, X_{1}^{b}\right)$. The cumulative state can be represented $U\left(X_{1}^{a}, X_{1}^{b}\right) U\left(X_{3}^{a}, X_{2}^{b}\right)^{-1}$ $\left|\psi_{3}\right\rangle=c_{1132} U\left(\left(X_{1}^{a}, X_{1}^{b}\right) \oplus\left(X_{3}^{a}, X_{2}^{b}\right)\right)^{-1}\left|\psi_{3}\right\rangle$ or $U\left(X_{1}^{a}, X_{1}^{b}\right) U\left(X_{2}^{a}, X_{3}^{b}\right)^{-1}\left|\psi_{2}\right\rangle=$ $c_{1123} U\left(\left(X_{1}^{a}, X_{1}^{b}\right) \oplus\left(X_{2}^{a}, X_{3}^{b}\right)\right)^{-1}\left|\psi_{2}\right\rangle$, where $\left|c_{1123}\right|=\left|c_{1132}\right|=1$, depending on whether terminal 2 or 3 measures $(a)$ or $(b)$. If we are to consider the clockwise cyclicity and the indices 'wrapping around' (with $X_{4}=X_{1}$ ), we have the transformation $U\left(\left(X_{i}^{a}, X_{i}^{b}\right) \oplus\left(X_{i+1}^{a}, X_{i+2}^{b}\right)\right)^{-1}$. To be able to recover the state at the receiving terminals, we formulate a combination of classical bits $X_{1}^{a}, X_{1}^{b}, X_{2}^{a}, X_{2}^{b}, X_{3}^{a}$ and $X_{3}^{b}$ to obtain an inverse unitary transformation for recovering the states. For the scheme with three users and three 3-qubit GHZ states, we use four central channels: $E=\left(\sum_{\oplus i=1}^{3} X_{i}^{(a)}, \sum_{\oplus i=1}^{3} X_{i}^{(b)}\right)$ and $E_{j}=\left(\sum_{\oplus i=1 \backslash j}^{3} X_{i}^{(a)}, \sum_{\oplus i=1 \backslash j}^{3} X_{i}^{(b)}\right)$. Classical information is transmitted using these four central channels to the three users, upon which the inverse transformation can be applied to the quantum state received at the terminal to get the cross-transmitted states.

This proposal can be generalised to the case of $n$-qubits by considering an $n$ qubit GHZ state: $|\xi\rangle=\frac{1}{\sqrt{2}}\left(\left|0^{\otimes n}+\right| 1^{\otimes n}\right)$. In this case, there will be $n+1$ channels with $E=\left(\sum_{\oplus i=1}^{n} X_{i}^{(a)}, \sum_{\oplus i=1}^{n} X_{i}^{(b)}\right)$ and $E_{j}=\left(\sum_{\oplus i=1 \backslash j}^{n} X_{i}^{(a)}, \sum_{\oplus i=1 \backslash j}^{n} X_{i}^{(b)}\right)$. As in the case of the two parties, perfect transmission of quantum states is impossible without prior entanglement. Fidelity Analysis Considering the quantum Fano inequality [10,11]: $H_{T W} \leq \eta\left(f_{e}\right)$, where $H_{T W}$ is the entropy of the twirling channel, $f_{e}$ is the entanglement fidelity and $\eta(x)=-x \log _{2} x-(1-x) \log _{2} \frac{(1-x)}{3}$. Let $R_{i}$ be the reference system of $A_{i}$. Since $H\left(R_{i}\right)=2 \forall i$, the upper bound for the sum of mutual information is given by [11,12]: $I\left(R_{i}: U_{i}\right) \leq 2-I\left(R_{i}: B_{i}\right) \forall i$ with the input being $A_{i}$ and output $U_{i} \otimes B_{i}$. If we have no prior entanglement, the systems $R_{i} U_{i} D_{i} \forall i$ are independent. In the case of the butterfly network with 
three nodes and three 3-qubit GHZ resource states, we have four central channels: $F_{i}=\left\{E, E_{1}, E_{2}, E_{3}\right\}$. When $F_{i} \forall i$ is a two-bit classical channel, the relation $H\left(R_{1} R_{2} R_{3} U_{1} U_{2} U_{3}\right) \leq H\left(F_{i} R_{1} R_{2} R_{3} U_{1} U_{2} U_{3}\right)$ holds. Using the Araki-Lieb inequality, we see $I\left(R_{1} R_{2} R_{3}: B_{1} B_{2} B_{3}\right) \leq 9-\sum_{i=1}^{3} I\left(R_{i}: B_{i}\right)$.

If we focus on the twirling of the channels and the transmission information as $I_{T W}\left(R_{i}: B_{i}\right)$ for single channels and $I_{T W}\left(R_{1} R_{2} R_{3}: B_{1} B_{2} B_{3}\right)$ for all taken together, and considering that entanglement fidelity remains unchanged upon twirling as well as writing $H_{T W}\left(R_{1} R_{2} R_{3} U_{1} U_{2} U_{3}\right)=\sum_{i=1}^{3} H_{T W}\left(R_{i} U_{i}\right)$, we obtain: $\sum_{i=1}^{3} H_{T W}\left(R_{i} U_{i}\right) \geq \frac{3}{2}$. Using, the quantum Fano inequality and the relation of the entropy and entanglement fidelity, $H_{T W}(R B) \leq \eta\left(f_{e}\right)$, where $\eta(x)=-x \log _{2} x-$ $(1-x) \log _{2} \frac{1-x}{3}$. Using this expression in the relation between twirled entropy and entropy fidelity, we have $\sum_{i=1}^{3} \eta\left(f_{e, i}\right) \geq \frac{3}{2}$. For the $n$-qubit case, if we use the convexity of $\eta$, we have $\eta\left(\sum_{i=1}^{n} f_{e, i}\right) \geq \frac{n}{2}$. Solving this inequality and using the relationship between average $f_{i}$ and $f_{e, i}$ [6]: $\frac{1+n f_{e, i}}{n+1}$, gives us $\sum_{i=1}^{n} f_{i} \leq \frac{2.8512 n}{n+1}$, where $f_{i}$ is the average entanglement fidelity of the $i^{\text {th }}$ channel and $n$ is the number of nodes in the quantum network. The operation of this higher-order butterfly scheme cannot be undertaken without prior entanglement.

Example: Let us say we have the state $\left|\psi_{1}\right\rangle=\alpha_{1}|0\rangle+\beta_{1}|1\rangle$ at terminal $A_{1}$ and we want to transfer it to node $B_{2}$. We then use the first GHZ state $\left|\phi_{1}\right\rangle=\frac{1}{\sqrt{2}}(|000\rangle+$ $|111\rangle)_{(1,1),(2,1),(3,1)}$. We undertake a Bell state measurement on $A_{1}$ and $A_{1,1}$. Let us say we measure the state $\left|\phi_{+}\right\rangle=\frac{1}{\sqrt{2}}(|01\rangle+|10\rangle)$ which corresponds to $X_{1}^{(a)}=(1,0)$. We undertake a single qubit measurement in the $| \pm\rangle$ basis, and let us say we have the third node $A_{3}$ measure $X_{1}^{(b)}=1$ corresponding to $\frac{1}{\sqrt{2}}(|0\rangle-|1\rangle)$. Since the other nodes have no qubits to transmit, they insert 1 for all other variables: $X_{2}^{(a)}=X_{3}^{(a)}=X_{1}^{(b)}=$ $X_{2}^{(b)}=1$. The nodes transmit $\mathcal{A}_{1}=1, \mathcal{A}_{2}=0$ and $\mathcal{A}_{3}=0$ for the activation variables, and $\mathcal{C}_{1}=0$ for the relevant cyclicity variable to denote clockwise movement in the network. Moving through the network, we obtain the relevant classical information on the output nodes. Then, using $\sigma_{x} \sigma_{z}$ on the second node $B_{2}$, we can obtain the arbitrary input state at this node.

The $n$-qubit GHZ states can be used for network recovery after multinode failure for an arbitrary graph state using tessellation of such GHZ states in partitioned blocks of the graph, as shown in Fig. 2a. The scheme for undertaking network recovery using the higher-order GHZ state-based network coding, formulated in the previous section, comprises of two distinct steps: Checking for operability of nodes and recovery of network operability. We prepare the graph state that constitutes the quantum network by initialising the states at the first terminal/node $|\phi\rangle$ and the other nodes with the state $|+\rangle=\frac{1}{\sqrt{2}}(|0\rangle+|1\rangle)$. We then operate with the CPHASE operation: $C Z_{i j}=$ $|0\rangle\left\langle\left. 0\right|_{i} I_{j}+\mid 1\right\rangle\left\langle\left. 1\right|_{i} Z_{j}\right.$, where $Z$ is the Pauli-Z matrix, between adjacent nodes. This gives us the entangled graph state which is the primary resource in the quantum network being studied.

For checking for operability of nodes, we use a hybrid classical-quantum approach. Classically, communication of node failure is done using 'pings' that are used in the ICMP echo protocol. However, this is not enough to ascertain quantum accessibility 

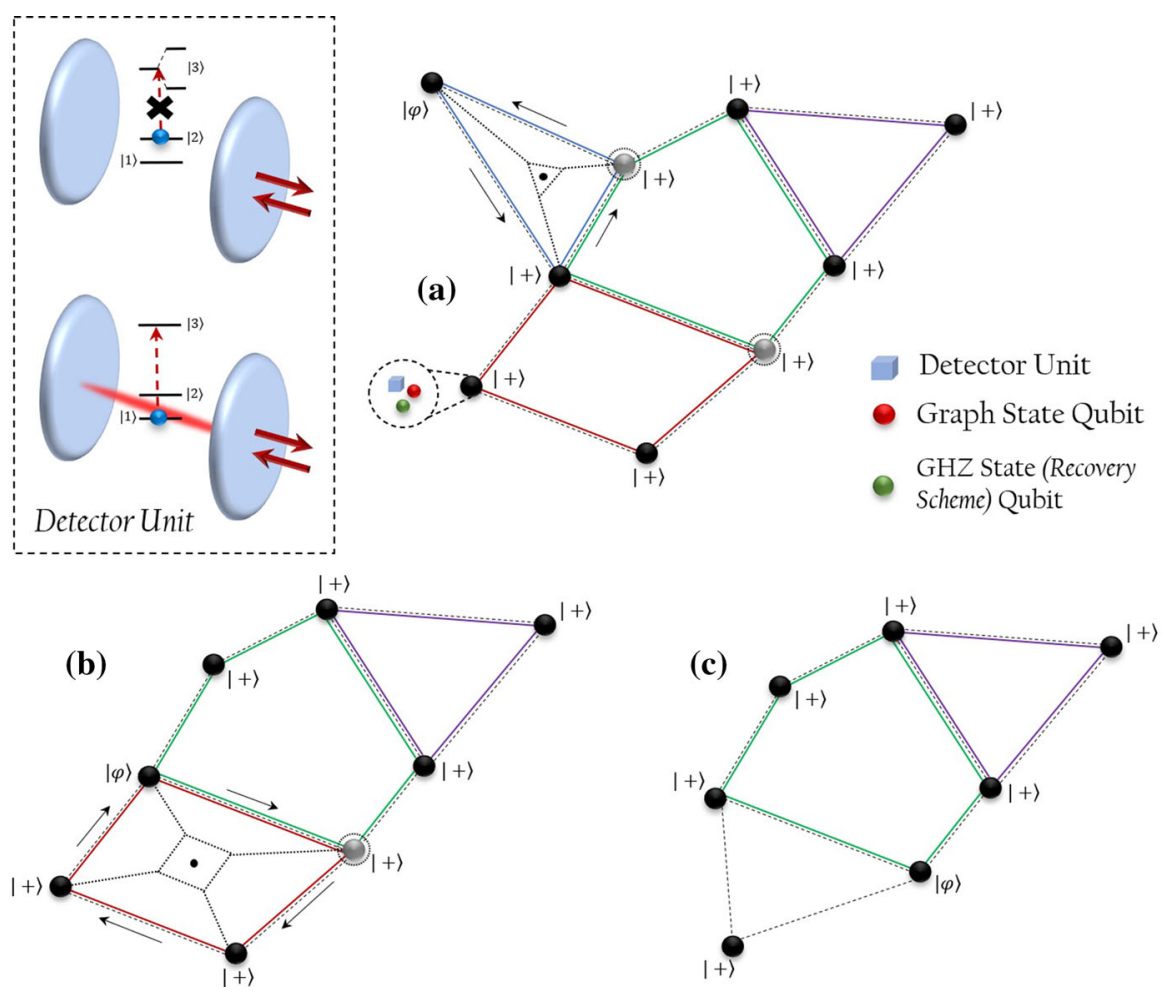

Fig. 2 Illustrative example for scheme for recovery of network operability from multinode failure: a The nodes begin with $|\phi\rangle$ at one node and $|+\rangle$ at the others, and we apply C-Phase operations on all the adjacent nodes. We then consider the possibility of two nodes failing (marked in grey). The coloured lines represent the GHZ states shared between adjacent nodes. a-c Mechanism for recovery upon multinode recovery of the network, using GHZ-based network coding in segments of the composite network. Inset is the detector unit comprising of an optical resonator with a three-level atom in a single-sided cavity.

and entanglement at the node. Additional to the classical connection, we need an additional layer to enable possibility to assess whether entanglement at the node is accessible and operative. This is done using quantum non-destructive measurements on the nodes of the graph, and any instance of node failure is relayed to the rest of the nodes in the affected subgraph using classical infrastructure. For optical systems, we can utilise non-destructive detection using an optical resonator containing a single atom prepared in a superposition of two states [13].

At each node, we position an optical resonator system with a three-level atom in a single-sided cavity, where one of the two mirrors is perfectly reflecting and there is a small transmission coefficient of the other mirror that allows for in- and outcoupling of light, which is an optical photon. Let the three states of the atom be tagged $\left|1_{a}\right\rangle$, $\left|2_{a}\right\rangle$ and $\left|3_{a}\right\rangle$. The cavity is designed such that it is overcoupled and resonant with the transition between the atomic states $\left|2_{a}\right\rangle$ and $\left|3_{a}\right\rangle$. If we prepare the atom initially in the state $\frac{\left|1_{a}\right\rangle+\left|2_{a}\right\rangle}{\sqrt{2}}$, an impinging photon makes it transform to $\frac{\left|2_{a}\right\rangle-\left|1_{a}\right\rangle}{\sqrt{2}}$ while it remains unchanged in the absence of an impinging photon. This is due to there being 
no interaction between the atom and photon when the atom is in the state $\left|1_{a}\right\rangle$ since any transition is far detuned, while when the atom is in the state $\left|2_{a}\right\rangle$, the strong photonatom coupling can lead to a normal-mode splitting and the photon undergoes reflection without entering the cavity. We can measure this phase flip using a $\frac{\pi}{2}$ rotation map to map $\frac{\left|1_{a}\right\rangle+\left|2_{a}\right\rangle}{\sqrt{2}} \rightarrow\left|1_{a}\right\rangle$ and $\frac{\left|1_{a}\right\rangle-\left|2_{a}\right\rangle}{\sqrt{2}} \rightarrow\left|2_{a}\right\rangle$, and then cavity-enhanced fluorescence state detection can be used to distinguish between the states $\left|2_{a}\right\rangle$ and $\left|1_{a}\right\rangle$ [14].

The operative interaction in such an optical resonator system is based on the principles of cavity quantum electrodynamics, which is applicable to various physical systems and is particularly robust [15]. In the physical realisation of such a system undertaken by Reiserer-Ritter-Rempe [13], a single ${ }^{87} \mathrm{Rb}$ is trapped in an optical lattice that is placed at the centre of a Fabry Perot resonator. The transmission of the coupling mirror was taken as $95 \mathrm{ppm}$ - which is much larger than that of the high reflector as well as the absorption and scattering losses, which happened to be around $8 \mathrm{ppm}$. In this physics realisation, the cavity field decay rate is taken to be $15.7 \mathrm{MHz}$, the atomic dipole decay rate is $18.8 \mathrm{MHz}$ and the measured atom-cavity coupling constant on the $\left|2_{a}\right\rangle \leftrightarrow\left|3_{a}\right\rangle$ is $42.1 \mathrm{MHz}$ [16], which means that the system operates in the strongcoupling regime of cavity quantum electrodynamics [17]. With the aforementioned parameters, the probability to detect a single input photon without post-selection on reflection from the cavity is calculated to be $74 \%$ [13].

Upon the non-destructive detection of a lost photon, the classical infrastructure used for classical communication in our model for higher-order butterfly networks is used to alert the other nodes in the subnetworks of which the failed node(s) is(are) a part of. This is undertaken using a parity code check. This comprises of $E=\left(\sum_{\oplus i=1}^{n} X_{i}^{(a)}, \sum_{\oplus i=1}^{n} X_{i}^{(b)}\right)$ and $E_{j}=\left(\sum_{\oplus i=1 \backslash j}^{n} X_{i}^{(a)}, \sum_{\oplus i=1 \backslash j}^{n} X_{i}^{(b)}\right)$ for $n$ nodes of a subnetwork. However, unlike in the case of the higher-order butterfly network, we do not send any single- or two-qubit measurements over these channels but rather if the check on each node yields a success (' 1 ') or failure (' 0 '). In this manner, each node in the subnetwork can exactly know which node(s) is(are) non-operative. Upon ascertaining the same, we operate with a $\sigma_{z}$ to remove the particular failed node(s) from the graph state.

For recovery of network operability, we can use the network coding formalism for higher-order butterfly networks developed and discussed in the previous section. In this framework, the key point is to tessellate the complex multiqubit network with subnetworks comprising of $n_{i}$ qubit GHZ state for the $i^{\text {th }}$ subnetwork comprising of $n_{i}$ nodes, along with the requisite classical infrastructure, as highlighted in the model formulated for higher-order butterfly networks. As soon as we ascertain which nodes are non-operative, we can use the GHZ state in the subnetwork to replace the failed node, as shown in Fig. 2a-c. Failure of all nodes that a subnetwork shares with the rest of the network, along with both additional qubits on either side of this set precipitates a point of criticality wherein the network operability cannot be recovered using the formulated scheme. The recovery of rate-optimal coded graph state networks can also be realised using modified graph-state codes [18].

In this letter, we have formulated and constructed a higher-order butterfly network using a multiqubit GHZ states. We studied the fidelity bounds of transmission in such GHZ-based higher-order butterfly networks that scale as $\frac{n}{n+1}$ for the number of 
terminals, which we use to construct a scheme for quantum network recovery from multinode failure. This is particularly useful for making quantum networks resilient against failures at multiple nodes.

Data Availability Data sharing is not applicable to this article as no new data were created or analysed in this study.

\section{References}

1. Gisin, N., Thew, R.: Quantum communication. Nat. Photonics 1(3), 165-171 (2007)

2. Mattle, K., et al.: Dense coding in experimental quantum communication. Phys. Rev. Lett. 76(25), 4656 (1996)

3. Pan, J.-W., et al.: Entanglement purification for quantum communication. Nature 410(6832), 10671070 (2001)

4. Harrow, A., Hayden, P., Leung, D.: Superdense coding of quantum states. Phys. Rev. Lett. 92(18), $187901(2004)$

5. Vaidman, L.: Teleportation of quantum states. Phys. Rev. A 49(2), 1473 (1994)

6. Hayashi, M., et al.: 'Quantum network coding. In: Annual Symposium on Theoretical Aspects of Computer Science. Springer, Berlin (2007)

7. Ahlswede, R., et al.: Network information flow. IEEE Trans. Inf. Theory 46(4), 1204-1216 (2000)

8. Iwama, K., et al.: 'Quantum network coding for general graphs. arXiv preprint arXiv:quant-ph/0611039 (2006)

9. Leung, D., Oppenheim, J., Winter, A.: Quantum network communication-the butterfly and beyond. IEEE Trans. Inf. Theory 56(7), 3478-3490 (2010)

10. Sharma, N.: Extensions of the quantum Fano inequality. Phys. Rev. A 78(1), 012322 (2008)

11. Hayashi, M.: Prior entanglement between senders enables perfect quantum network coding with modification. Phys. Rev. A 76(4), 040301 (2007)

12. Imai, H., et al.: A quantum information theoretical model for quantum secret sharing schemes. arXiv preprint arXiv:quant-ph/0311136 (2003)

13. Reiserer, A., et al.: A quantum gate between a flying optical photon and a single trapped atom. Nature 508(7495), 237-240 (2014)

14. Bochmann, J., et al.: Lossless state detection of single neutral atoms. Phys. Rev. Lett. 104(20), 203601 (2010)

15. Duan, L.-M., Kimble, H.J.: Scalable photonic quantum computation through cavity-assisted interactions. Phys. Rev. Lett. 92(12), 127902 (2004)

16. Reiserer, A., et al.: Ground-state cooling of a single atom at the center of an optical cavity. Phys. Rev. Lett. 110(22), 223003 (2013)

17. Kleppner, D.: Cavity quantum electrodynamics. In: International Quantum Electronics Conference. Optical Society of America (1986)

18. Nadkarni, P., Raina, A., Garani, S.S.: Modified graph-state codes for single-node recovery in quantum distributed storage. Phys. Rev. A. 102, 062430 (2020)

Publisher's Note Springer Nature remains neutral with regard to jurisdictional claims in published maps and institutional affiliations. 